\title{
Differential Equation of the Loxodrome on a Helicoidal Surface
}

\author{
Murat Babaarslan $^{1}$ and Yusuf Yayli ${ }^{2}$ \\ ${ }^{1}$ (Department of Mathematics, Bozok University, 66100, Yozgat, Turkey) \\ ${ }^{2}$ (Department of Mathematics, Ankara University, 06100, Ankara, Turkey)
}

(E-mail: murat.babaarslan@bozok.edu.tr)

\begin{abstract}
In nature, science and engineering, we often come across helicoidal surfaces. A curve on a helicoidal surface in Euclidean 3-space is called a loxodrome if the curve intersects all meridians at a constant azimuth angle. Thus loxodromes are important in navigation. In this paper, we find the differential equation of the loxodrome on a helicoidal surface in Euclidean 3-space. Also we give some examples and draw the corresponding pictures via the Mathematica computer program to aid understanding of the mathematics of navigation.
\end{abstract}

\section{KEYWORDS}

1. Loxodrome. 2. Helicoidal Surface. 3. Euclidean Space.

Submitted: 8 August 2014. Accepted: 24 February 2015. First published online: 27 April 2015.

1. INTRODUCTION. We can define many well known objects with respect to making a constant angle with a distinct direction. Our first example is helices that are special curves that lie on helicoids (minimal surfaces), see Figure 4. These are characterised by the property that tangent lines make a constant angle with a fixed direction. Helices are geodesics or length minimizing curves on the helicoids (Papst, 2011). A generalisation of geodesics is magnetic curves. In Physics, a magnetic curve represents a trajectory of a charged particle moving on the manifold under the action of a magnetic field. Thus helices in Euclidean Space (or $\mathbb{E}^{3}$ ) are also characterised by the following property: they are magnetic trajectories related to magnetic fields parallel to their axis (Munteanu, 2013). There are many interesting applications of helices such as $\alpha$-helices, K-helices, Deoxyribonucleic Acid (DNA) double helix, collagen triple helix, nanosprings, carbon nanotubes, etc. (Ilarslan and Boyacioglu, 2008). A further example is logarithmic spirals or spira mirabilis, extensively investigated by Jacob Bernoulli, which make a constant angle with the radial direction. One of their interesting properties is the fact that they are self-similar. Thus we can see them in some phenomena and on different objects around us, for example; the approach of a hawk to its prey, the approach of an insect to a light source, the arms of a spiral galaxy, the nerves of the cornea, some spiral roses, sunflower heads, shells, horns, cosmology, fractal structures, architectures and so on (Boyadzhiev, 1999, 2007; Munteanu, 2010). 

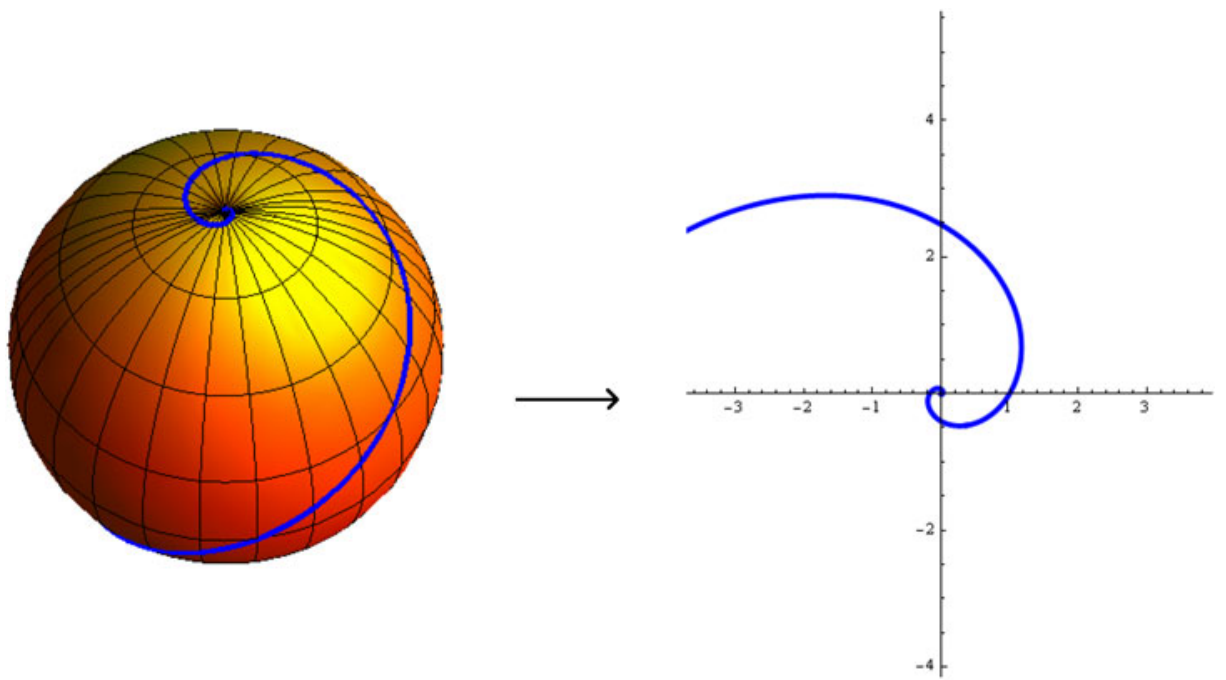

Figure 1. Stereographic projection of a loxodrome on a sphere (Babaarslan and Munteanu, 2013).

The third famous example is loxodromes or rhumb lines which intersect the meridians at a constant angle on the Earth's surface. Generally loxodromes are not great circles (geodesics), see Tseng and Chang (2014). Hence they do not measure the shortest distance between two points on the Earth's surface. However loxodromes are primarily used in navigation since they do not require a change of course, i.e., course $=\theta=$ constant (Kos et al., 2009). In 1569, a major development for navigation was the Mercator map. The Mercator projection was used for centuries, but unfortunately has navigational errors (Alexander, 2004; Babaarslan and Munteanu, 2013). It is well known that by using stereographic projection, loxodromes on a sphere are projected as logarithmic spirals on the plane of the equator (Noble, 1905), see Figure 1. Also, if they are plotted on the Mercator grid, then they have the form of straight lines. For example; a voyager can head at a constant bearing $73^{\circ}$ East of North to travel from New York to London (Alexander, 2004). Mercator used a conformal map in order for loxodromes on a sphere to be drawn as straight lines on a plane (Babaarslan and Munteanu, 2013).

Noble (1905) found the equations of loxodromes on the rotational surfaces and also gave some particular examples of them such as sphere and spheroid. Kos et al. (1999, 2009) computed the arc-length of the loxodrome on a sphere. Also Petrovic (2007) determined the arc-length of the loxodrome on a spheroid. Babaarslan and Munteanu (2013) computed all time-like loxodromes on the rotational surfaces which have time-like meridians or space-like meridians, respectively in Minkowski 3-space. Similarly, Babaarslan and Yayli (2014) found all space-like loxodromes on the rotational surfaces in Minkowski 3-space and gave some examples using the Mathematica computer programme.

We know that helicoidal surfaces are the natural generalisations of rotational surfaces. Furthermore we often come across helicoidal structures and objects that are related to navigation in nature, science and engineering. For instance; creeper plants, helicoidal staircases, helicoidal conveyors, parking garage ramps, helicoidal railways, moving walkways and footbridges, helicoidal towers and (nowadays) skyscrapers 
(Capanna et al., 2012), helicoidal fractures in geology (Pollard and Fletcher, 2005), helicoidal paths, tropical cyclones (Levina and Montgomery, 2010), electronic states on the helicoidal surfaces (Jensen, 2009) and helical channels (Morales and Rosa, 2007).

According to the authors' knowledge, there is no article related to equation of loxodrome on a helicoidal surface in Euclidean 3-space. The main goal of this paper is to find the differential equation of the loxodrome on a helicoidal surface in Euclidean 3space. Also we give some examples and draw the corresponding pictures by using the Mathematica computer program to understand loxodromes better. We hope to inspire more people to research the relations between loxodromes on different structures and other branches of science.

2. PRELIMINARIES. In this section, we recall some important notions and also give some properties of curves and surfaces in Euclidean 3 -space $\mathbb{E}^{3}$. For more details, we refer to Babaarslan and Munteanu (2013), Babaarslan and Yayli (2014), Guler et al. (2010), Ikawa (2000), O’Neill (1966) and Do Carmo (1976).

The inner product of two vectors $x=\left(x_{1}, x_{2}, x_{3}\right)$ and $y=\left(y_{1}, y_{2}, y_{3}\right)$ in $\mathbb{E}^{3}$ is given by:

$$
\langle x, y\rangle=x_{1} y_{1}+x_{2} y_{2}+x_{3} y_{3}
$$

The norm (length) of a vector $x \in \mathbb{E}^{3}$ is $\|x\|=\sqrt{\langle x, x\rangle}$ and it is called a unit vector if $\|x\|=1$. The angle $\theta$ between $x$ and $y$ is given by

$$
\cos \theta=\frac{\langle x, y\rangle}{\|x\|\|y\|}
$$

where $0<\theta<\pi$. In particular, if $\theta=\pi / 2$, then $\langle x, y\rangle=0$. Thus we can define vectors to be orthogonal provided their inner product is zero.

Let $\beta: I \subset \mathbb{R} \rightarrow \mathbb{E}^{3}$ be a regular curve in $\mathbb{E}^{3}$ (i.e., $\dot{\beta} \neq 0$ for all $t \in I$ ). The arc-length of $\beta$ between $t_{0}$ and $t$ is given by

$$
s(t)=\int_{t_{0}}^{t}\|\dot{\beta}(t)\| d t
$$

Then the parameter $s \in J \subset \mathbb{R}$ is determined such as $\left\|\beta^{\prime}(s)\right\|=1$. Thus $\beta$ is called a unit speed curve if $\left\|\beta^{\prime}(s)\right\|=1$.

Now we give the definitions of rotational surfaces and helicoidal surfaces and also some important formulas in $\mathbb{E}^{3}$.

Let $\beta: I \subset \mathbb{R} \rightarrow P$ be a regular curve in a plane $P \subset \mathbb{E}^{3}$ and $l$ be a straight line in $P$. If this profile curve $\beta$ is rotated about the axis $l$, then it sweeps out a rotational surface in $\mathbb{E}^{3}$. Similarly, we assume that when the profile $\beta$ rotates about the axis $l$, it simultaneously displaces parallel to $l$ so that the speed of displacement is proportional to the speed of rotation. As a result it sweeps out a helicoidal surface in $\mathbb{E}^{3}$.

Let us assume that $l=\operatorname{span}\{(0,0,1)\}$. Then the rotation which leaves the axis $l$ invariant is given by the following rotational matrix

$$
\left[\begin{array}{ccc}
\cos v & -\sin v & 0 \\
\sin v & \cos v & 0 \\
0 & 0 & 1
\end{array}\right], v \in I \subset \mathbb{R}
$$

If the rotation axis is $l$, then there is a Euclidean transformation where the axis $l$ is transformed to the $x_{3}$-axis of $\mathbb{E}^{3}$. Rotation leaves the planes that are orthogonal to 
the rotation axis $l$ invariant. Thus we can choose the profile curve in a plane containing the rotation axis $l$. As a result we can take the profile curve $\beta$ in the $\left(x_{1} x_{3}\right)$-plane that can be parameterised by $\beta(u)=(f(u), 0, g(u)), u \in I \subset \mathbb{R}$, where $f(u) \neq 0$.

A helicoidal surface with the rotation axis $l$ and the pitch $\lambda \in \mathbb{R} \backslash\{0\}$ can be parameterised as follows

$$
H(u, v)=\left[\begin{array}{ccc}
\cos v & -\sin v & 0 \\
\sin v & \cos v & 0 \\
0 & 0 & 1
\end{array}\right]\left[\begin{array}{c}
f(u) \\
0 \\
g(u)
\end{array}\right]+\lambda v\left[\begin{array}{l}
0 \\
0 \\
1
\end{array}\right]
$$

or

$$
H(u, v)=(f(u) \cos v, f(u) \sin v, g(u)+\lambda v)
$$

When $g$ is a constant function, the helicoidal surface is called the right helicoidal surface. Also, when $\lambda=0$, the helicoidal surfaces reduce to rotational surfaces.

For a surface $X(u, v)$, the first fundamental form or line-element in the base $\left\{X_{u}, X_{v}\right\}$ is given by

$$
d s^{2}=E d u^{2}+2 F d u d v+G d v^{2}
$$

where $E=\left\langle X_{u}, X_{u}\right\rangle, F=\left\langle X_{u}, X_{v}\right\rangle$ and $G=\left\langle X_{v}, X_{v}\right\rangle$ are the coefficients of the first fundamental form.

The angle $\theta$ between two regular curves $\beta: I \subset \mathbb{R} \rightarrow X$ and $\gamma: I \subset \mathbb{R} \rightarrow X$ which intersect at $t=t_{0}$ is given by

$$
\cos \theta=\frac{\left\langle\dot{\beta}\left(t_{0}\right), \dot{\gamma}\left(t_{0}\right)\right\rangle}{\left\|\dot{\beta}\left(t_{0}\right)\right\|\left\|\dot{\gamma}\left(t_{0}\right)\right\|}
$$

In particular, the angle $\varphi$ between the constant parameter curves of $X(u, v)$ is given by

$$
\cos \varphi=\frac{\left\langle X_{u}, X_{v}\right\rangle}{\left\|X_{u}\right\|\left\|X_{v}\right\|}=\frac{F}{\sqrt{E G}}
$$

Thus the constant parameter curves are orthogonal if and only if $F(u, v)=0$ for all $(u, v)$.

3. DIFFERENTIAL EQUATION OF THE LOXODROME. Let us consider the helicoidal surface $H$ that is given by Equation (5). To simplify the calculations, we assume that $f^{\prime 2}(u)+g^{\prime 2}(u)=1$ for all $u \in J \subset \mathbb{R}$, that is, the profile curve $\beta$ is parameterised by arc-length parameter. The coefficients of first fundamental form of the helicoidal surface are

$$
E=\left\langle H_{u}, H_{u}\right\rangle=1, F=\left\langle H_{u}, H_{v}\right\rangle=\lambda g^{\prime}(u) \text { and } G=\left\langle H_{v}, H_{v}\right\rangle=f^{2}(u)+\lambda^{2}
$$

Obviously, the constant parameter curves of $H(u, v)$ are orthogonal if and only if $H(u, v)$ is either a right helicoidal surface or a rotational surface. Substituting Equation (9) into (6), the first fundamental form of the helicoidal surface is determined by the following equation

$$
d s^{2}=d u^{2}+2 \lambda g^{\prime}(u) d u d v+\left(f^{2}(u)+\lambda^{2}\right) d v^{2}
$$


Also the arc-length of any curve on the helicoidal surface between $u_{1}$ and $u_{2}$ is given by

$$
s=\int_{u_{1}}^{u_{2}} \sqrt{1+2 \lambda g^{\prime}(u) \frac{d v}{d u}+\left(f^{2}(u)+\lambda^{2}\right)\left(\frac{d v}{d u}\right)^{2}} d u
$$

We may assume that the loxodrome $\alpha(t)$ is the image of a curve $(u(t), v(t))$ in the $(u v)$ plane by $H$. Since in the basis $\left\{H_{u}, H_{v}\right\}$, the vector $\alpha^{\prime}(t)$ has the coordinates $\left(u^{\prime}, v^{\prime}\right)$ and the vector $H_{u}$ has the coordinates $(1,0)$. At the point $H(u, v)$ where the loxodrome intersects the meridian at a constant azimuth $\theta$, we have

$$
\begin{aligned}
\cos \theta=\frac{\left\langle\alpha^{\prime}(t), H_{u}\right\rangle}{\left\|\alpha^{\prime}(t)\right\|\left\|H_{u}\right\|} & =\frac{E d u+F d v}{\sqrt{E^{2} d u^{2}+2 E F d u d v+E G d v^{2}}} \\
& =\frac{d u+\lambda g^{\prime}(u) d v}{\sqrt{d u^{2}+2 \lambda g^{\prime}(u) d u d v+\left(f^{2}(u)+\lambda^{2}\right) d v^{2}}}
\end{aligned}
$$

From Equation (12), we obtain the following differential equation of the loxodrome on the helicoidal surface:

$$
\left(\cos ^{2} \theta\left(f^{2}(u)+\lambda^{2}\right)-\lambda^{2} g^{\prime 2}(u)\right)\left(\frac{d v}{d u}\right)^{2}-2 \lambda \sin ^{2} \theta g^{\prime}(u) \frac{d v}{d u}=\sin ^{2} \theta
$$

Thus the general solution of the differential equation of the loxodrome on the helicoidal surface is

$$
v=\int_{u_{0}}^{u} \frac{2 \lambda \sin ^{2} \theta g^{\prime}(u)+\varepsilon \sqrt{\sin ^{2} 2 \theta\left(f^{2}(u)-\lambda^{2}\left(g^{\prime 2}(u)-1\right)\right)}}{2 \cos ^{2} \theta\left(f^{2}(u)+\lambda^{2}\right)-2 \lambda^{2} g^{\prime 2}(u)} d u
$$

where $\varepsilon= \pm 1$.

For the loxodrome on the right helicoidal surface, Equation (13) reduces to Equation (15):

$$
\cos ^{2} \theta\left(f^{2}(u)+\lambda^{2}\right)\left(\frac{d v}{d u}\right)^{2}=\sin ^{2} \theta
$$

With the solution of this differential equation, we find the general solution of the differential equation of the loxodrome on the right helicoidal surface as

$$
v=\varepsilon \tan \theta \int_{u_{0}}^{u} \frac{d u}{\sqrt{f^{2}(u)+\lambda^{2}}}
$$

where $\varepsilon= \pm 1$.

Noble (1905) and Kos et al. (2009) found the differential equation of the loxodrome on a rotational surface. If we take $\lambda=0$ in Equation (16), we find the general solution of the differential equation of the loxodrome on rotational surface as follows

$$
v=\varepsilon \tan \theta \int_{u_{0}}^{u} \frac{d u}{f(u)}
$$

where $\varepsilon= \pm 1$. This equation coincides with the equation in (Noble, 1905; Kos et al., 2009). 


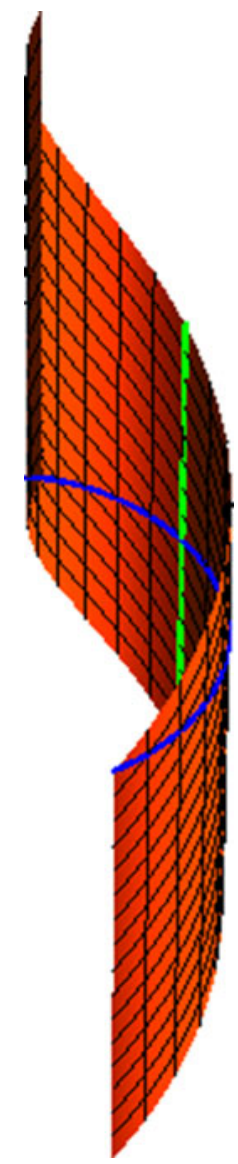

Figure 2. The helicoidal surface; loxodrome (blue), meridian (green).

Next, we give the arc-length of the loxodrome on the right helicoidal surface. The arc-length of any curve on the right helicoidal surface between $u_{1}$ and $u_{2}$ is given by

$$
s=\int_{u_{1}}^{u_{2}} \sqrt{1+\left(f^{2}(u)+\lambda^{2}\right)\left(\frac{d v}{d u}\right)^{2}} d u
$$

By using Equation (15), the arc-length of the loxodrome is defined as follows

$$
s=\frac{u_{2}-u_{1}}{\cos \theta}
$$

Similarly, the arc-length of the loxodrome on the rotational surface coincides with Equation (19).

Now we give three examples of loxodromes that lie on the helicoidal surfaces to strengthen our main results.

Example 1. Let us take $f(u)=2, g(u)=u+1, u \in(-5,5), \lambda=3, \theta=\pi / 3, \varepsilon=1$ and $v(0)$ $=0$. By the straightforward calculation, we have $v \in(-2 \cdot 70959,2 \cdot 70959)$. Also the arclength of the loxodrome is equal to $7 \cdot 50903$. We can draw the helicoidal surface $H(u$, $v)$, the meridian $(v=$ constant $)$ and the loxodrome $H(u, v(u))$ in Figure 2. 


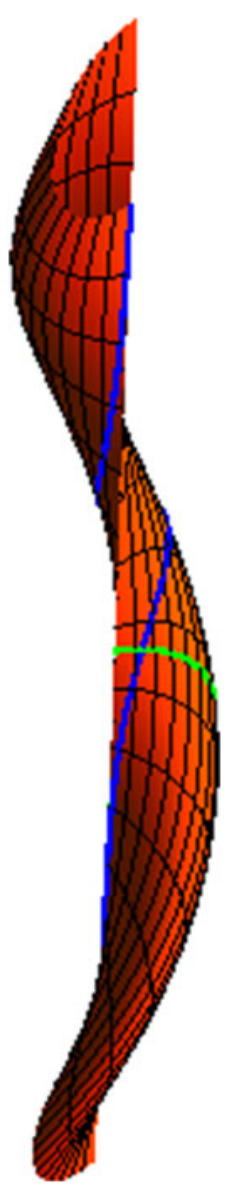

Figure 3. The helicoidal surface; loxodrome (blue), meridian (green).

Example 2. Let us take $f(u)=\sin u, g(u)=\cos u, u \in(0 \cdot 01,3 \cdot 14), \lambda=2, \theta=\pi / 2, \varepsilon=$ -1 and $u_{0}=1.5708$. By the straightforward calculation, we have $v \in(-2.64916$, $3 \cdot 56775)$. The arc-length of the loxodrome is equal to 11.5391 . We can draw the helicoidal surface $H(u, v)$, the meridian $(v=$ constant $)$ and the loxodrome $H(u, v(u))$ in Figure 3.

Due to the energy minimisation principle, minimal surfaces are omnipresent in nature and science. Thus their study has been a fascinating notion for centuries. One of the non-trivial examples of minimal surfaces is the helicoid found by Meusnier (Bates et al., 2008). We can construct it by using Equation (5). The parallel $(u=$ constant $)$ on the helicoid is a helix and it is perpendicular to the meridians ( $v=$ constant). Thus a helix on the helicoid is also a loxodrome. This is an important property since helices are strongly related to navigation. Thus we can give the following example: 


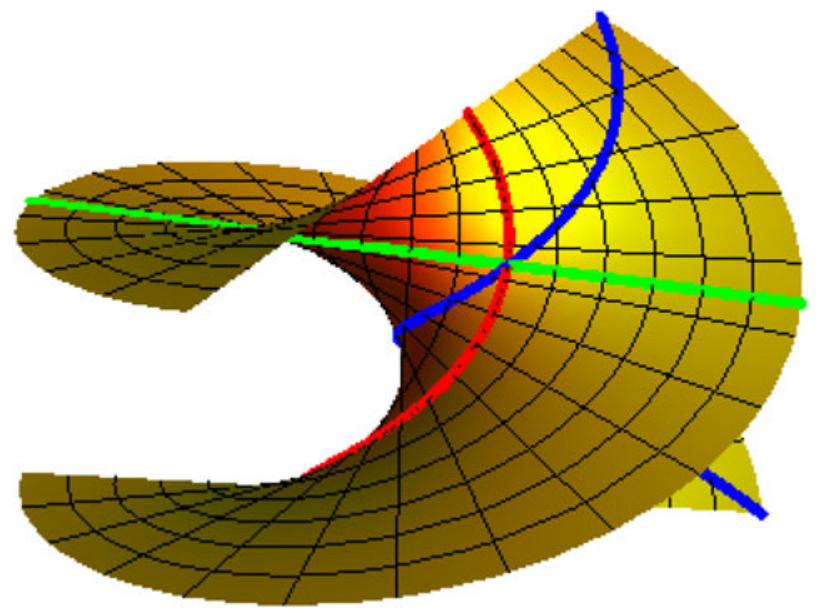

Figure 4. The right helicoidal surface (helicoid); loxodrome (blue), meridian (green), parallel (red).

Example 3. Let us take $f(u)=u, g(u)=1, u \in(-4,4), \lambda=1, \theta=\pi / 4, \varepsilon=1$ and $v(0)=0$. By the straightforward calculation, we have $v \in(-2 \cdot 09,2 \cdot 09)$. Also the arc-length of the loxodrome is equal to $8 \sqrt{2}$. We can draw the right helicoidal surface (helicoid) $H(u, v)$, the meridian $(v=$ constant $)$, the parallel $(u=$ constant $)$ and the loxodrome $H$ $(u, v(u))$ in Figure 4.

4. CONCLUSION. Loxodromes correspond to curves that intersect all meridians at a constant angle on a helicoidal surface. Thus, they are very important in navigation. In general, the previous loxodrome studies were associated with rotational surfaces. But in nature, science and engineering, we can find surfaces other than rotational surfaces on which navigation is possible, for example; creeper plants, fractures in geology, parking garage ramps, helicoidal staircases, railways, moving walkways and footbridges, helical channels and so on. For this reason, in the present paper, we investigate the differential equations of loxodromes on the helicoidal surfaces in Euclidean 3space, hoping that many other researchers work on loxodromes on different structures. In future work, we will investigate the differential equations of loxodromes on the helicoidal surfaces in Minkowski 3-space since loxodromes in this space have important meaning in relativity theory and they are interesting from the points of view of geometric and mathematical cosmology (Babaarslan and Yayli, 2014).

\section{ACKNOWLEDGMENT}

The authors wish to express their gratitude to the reviewers for their valuable comments and suggestions.

\section{REFERENCES}

Alexander, J. (2004). Loxodromes: A Rhumb Way to Go. Mathematics Magazine, 77, 349-356.

Babaarslan, M., and Munteanu, M.I. (2013). Time-like loxodromes on rotational surfaces in Minkowski 3space. Annals of the Alexandru Ioan Cuza University-Mathematics, doi: 10.2478/aicu-2013-0021. (In press). 
Babaarslan, M., and Yayli, Y. (2014). Space-like loxodromes on rotational surfaces in Minkowski 3-space. Journal of Mathematical Analysis and Applications, 409, 288-298.

Bates, P.W., Wei, G.W., and Zhao, S. (2008). Minimal Molecular Surfaces and Their Applications. Journal of Computational Chemistry, 29, 380-391.

Boyadzhiev, K.N. (1999). Spirals and Conchospirals in the Flight of Insects. The College Mathematics Journal, 30, 23-31.

Boyadzhiev, K.N. (2007). Equiangular Surfaces, Self-Similar Surfaces, and the Geometry of Seashells. The College Mathematics Journal, 38, 265-271.

Capanna, A., Francaviglia, M., and Lorenzi, M.G. (2012), Architecture, Form, Expression. The Helicoidal Skyscrapers'Geometry. Proceedings of the 2012 Bridges Towson: Mathematics, Music, Art, Architecture, Culture. Towson University, Maryland, USA.

Do Carmo, M. P. (1976). Differential Geometry of Curves and Surfaces. Prentice Hall, Inc., Upper Saddle River, New Jersey.

Guler, E., Yayli, Y., and Hacisalihoglu, H.H. (2010). Bour's Theorem and the Gauss Map in 3-Euclidean Space. Hacettepe Journal of Mathematics and Statistics, 39, 515-525.

Ikawa, T. (2000). Bour's Theorem and Gauss Map. Yokohama Mathematical Journal, 48, 173-180.

Ilarslan, K., and Boyacioglu, Ö. (2008). Position vectors of a timelike and a null helix in Minkowski 3-space. Chaos, Solitons \& Fractals, 38, 1383-1389.

Jensen, B. (2009). Electronic states on the helicoidal surface. Physical Review A, 80, 022101.

Kos, S., Vranic, D., and Zec, D. (1999). Differential Equation of a Loxodrome on a Sphere. The Journal of Navigation, 52, 418-420.

Kos, S., Filjar, R., and Hess, M. (2009). Differential Equation of the Loxodrome on a Rotational Surface. Proceedings of the 2009 International Technical Meeting of The Institute of Navigation, Anaheim, CA.

Levina, G.V., and Montgomery, M.T. (2010). A First Examination of the Helical Nature of Tropical Cyclogenesis. Doklady Earth Sciences, 434, 1285-1289.

Morales, R.E.M., and Rosa, E.S. (2007). Modelling of Free Surface Flow in a Helical Channel with Finite Pitch. Journal of the Brazilian Society of Mechanical Sciences and Engineering, 29, 345-353.

Munteanu, M.I. (2010). From golden spirals to constant slope surfaces. Journal of Mathematical Physics, 51, 0735507.

Munteanu, M.I. (2013). Magnetic Curves in a Euclidean Space: One Example, Several Approaches. Publications De I'Institut Mathématique, 94, 141-150.

Noble, C.A. (1905). Note on loxodromes. Bulletin of the American Mathematical Society, 12, 116-119.

O’Neill, B. (1966). Elementary Differential Geometry, Academic Press Inc., New York.

Papst, I. (2011). A Biological Application of the Calculus of Variations. The Waterloo Mathematics Review, 1, 3-16.

Petrovic, M. (2007). Differential Equation of a Loxodrome on the Spheroid. International Journal of Maritime Science and Technology "Our Sea", 54, 87-89.

Pollard, D.D., and Fletcher, R.C. (2005). Fundamentals of Structural Geology. Cambridge University Press, United Kingdom.

Tseng, W-K., and Chang, W-J. (2014). Analogues between 2D Linear Equations and Great Circle Sailing. The Journal of Navigation, 67, 101-112. 